\title{
BARD1 variants Cys557Ser and Val507Met in breast cancer predisposition
}

Pia Vahteristo*,1,2 ${ }^{*}$ Kirsi Syrjäkoski ${ }^{3}$, Tuomas Heikkinen ${ }^{1}$, Hannaleena Eerola ${ }^{1,4}$, Kristiina Aittomäki ${ }^{5}$, Karl von Smitten ${ }^{6}$, Kaija Holli ${ }^{7}$, Carl Blomqvist ${ }^{4,8}$, Olli-Pekka Kallioniemi ${ }^{9}$ and Heli Nevanlinna ${ }^{1}$

\footnotetext{
${ }^{1}$ Department of Obstetrics and Gynecology, Helsinki University Central Hospital, Helsinki, Finland; ${ }^{2}$ Department of Medical Genetics, University of Helsinki, Helsinki, Finland; ${ }^{3}$ Laboratory of Cancer Genetics, Institute of Medical Technology, University of Tampere and Tampere University Hospital, Tampere, Finland; ${ }^{4}$ Department of Oncology, Helsinki University Central Hospital, Helsinki, Finland; ${ }^{5}$ Department of Clinical Genetics, Helsinki University Central Hospital, Helsinki, Finland; ${ }^{6}$ Department of Surgery, Helsinki University Central Hospital, Helsinki, Finland; ${ }^{7}$ Department of Oncology, University of Tampere and Tampere University Hospital, Tampere, Finland; ${ }^{8}$ Department of Oncology, Uppsala University Hospital, Uppsala, Sweden; ${ }^{9}$ Medical Biotechnology, VTT Technical Research Center of Finland and University of Turku, Turku, Finland
}

BARD1 (BRCA1-associated RING-domain 1) is a tumor suppressor whose protein product interacts with BRCA1, and in which rare somatic and germline mutations have been reported in breast, uterine, and endometrial cancers. We aimed to evaluate whether there are BARD1 genetic variants that contribute to breast cancer risk by screening the gene for germline alterations in 45 Finnish familial breast cancer patients and in seven patients with both breast and ovarian cancer. Two of the missense alterations identified (Cys557Ser and Val507Met) were recently suggested to associate with an increased breast cancer risk. We also analyzed these variants in large and independent series of familial and unselected breast cancer patients and healthy controls. No clearly deleterious mutations were detected in the initial mutation screening. No association of the Cys557Ser and breast cancer risk was observed as the variant was found altogether in $1.4 \%(16 / 1181)$ of familial and $2.2 \%(34 / 1565)$ of unselected breast cancer patients, and in $2.5 \%(27 / 1083)$ of healthy controls. The frequency of the Val-allele of the Val507Met variant was modestly higher among breast cancer patients than among healthy controls, although the difference did not reach statistical significance. No statistically significant association of the Cys557Ser or Val507Met variants with any clinicopathologic parameters was observed. These results suggest that the contribution of the BARD1 germline variants to breast cancer predisposition is very limited, and that neither Cys557Ser nor Val507Met have an effect on familial breast cancer susceptibility. European Journal of Human Genetics (2006) 14, 167-172. doi:10.1038/sj.ejhg.5201542; published online 7 December 2005

Keywords: BARD1; BRCA1 interacting protein; breast cancer risk; germline mutation

\footnotetext{
${ }^{*}$ Correspondence: Dr P Vahteristo, Department of Medical Genetics, Biomedicum Helsinki, PO Box 63, (Haartmaninkatu 8), FIN-00014 University of Helsinki, Helsinki, Finland. Tel: +358 91912 5600; Fax: + 35891912 5105; E-mail: pia.vahteristo@helsinki.fi Received 28 June 2005; revised 5 October 2005; accepted 21 October 2005; published online 7 December 2005
}

Introduction

Thus far, two highly penetrant breast and ovarian cancer susceptibility genes, BRCA1 and BRCA2, have been identified. Germline mutations in these genes are found in the majority of families (75-80\%) with both early-onset breast cancer and ovarian cancer, but in a considerably lower 
fraction of families with site-specific female breast cancer (no ovarian cancer or male breast cancer). ${ }^{1,2}$ Recently, the CHEK2 1100delC and Ile157Thr have been identified as new low-risk breast cancer susceptibility alleles. ${ }^{3-5}$ It has been suggested that a substantial proportion of the remaining familial aggregation of breast cancer might be attributed to several such low penetrance alleles that act multiplicatively, and thereby form a polygenic basis for breast cancer predisposition. 6,7

One potential candidate gene for breast cancer susceptibility is the BRCA1-associated RING-domain 1, BARD1 (GenBank U76638). The BARD1 protein was originally identified through a yeast two-hybrid screen using a BRCA1 RING domain as bait. ${ }^{8}$ The two proteins are structurally related: they both have an amino-terminal RING-domain and one (BRCA1) or two (BARD1) carboxyterminal BRCT domains. The RING domain mediates protein-protein interactions and may also participate in ubiquitination of the proteins, whereas the BRCT domain is found in a large number of proteins that predominantly participate in cell cycle checkpoint or DNA-repair pathways in response to DNA damage. These domains are evolutionarily well conserved, whereas the remaining of the sequences in both genes, with the exception of the ankyrin repeats of BARD1 that also participate in proteinprotein interactions, display a markedly lower degree of conservation.

The interaction between BRCA1 and BARD1 is mediated via their RING-finger motifs. ${ }^{8}$ The interaction is abolished by tumorigenic missense mutations in the RING domain of $B R C A 1$, implying that the formation of a stable complex between these proteins may be an essential aspect of BRCA1-mediated tumor suppression. ${ }^{8}$ A germline BARD1 mutation has also been reported in a patient diagnosed with breast, ovarian, and endometrial cancer, and somatic BARD1 mutations have been observed in breast and endometrial tumors; this suggests that BARD1 mutations might themselves be contributing factors in the development of some human carcinomas. ${ }^{9}$ This is further supported by the loss of the wild-type alleles both in the tumor tissue of the aforementioned patient with a germline mutation, as well as in the endometrial tumor with a somatic mutation. ${ }^{9}$ Functional analysis of the protein with a tumor-associated $B A R D 1$ germline mutation, Gln564His, has also shown that it is defective in p53-mediated apoptosis induction and results in reduced binding to polyadenylation factor CstF-50. ${ }^{10,11}$ Reduced BARD1 expression has also been observed in some sporadic breast tumors. ${ }^{12}$

As BARD1 is a plausible breast cancer susceptibility gene, we aimed to evaluate whether there are BARD1 genetic variants that contribute to breast cancer risk by screening the BARD1 gene for germline alterations among 45 Finnish $B R C A 1 / 2$ negative breast cancer families as well as in seven patients diagnosed with both breast and ovarian cancer.
During the course of this study, two of the identified BARD1 variants, Cys557Ser and Val507Met, were suggested to associate with breast cancer risk. Cys557Ser was suggested to increase familial breast cancer risk by fourfold, ${ }^{13}$ and the Val507Met was associated with a two-fold elevation in postmenopausal breast cancer risk. ${ }^{14}$ We evaluated these variants in extensive series of unselected and familial breast cancer patients, and healthy controls. We also investigated the possible association of the variants with breast tumor characteristics, age at cancer diagnosis, and survival.

\section{Patients and methods}

In the initial mutation analysis, the whole BARD1 coding region was screened in 45 families who have at least three first- or second-degree relatives, including the proband, affected with breast or ovarian cancer. The recruitment of the families, verification of the cancer diagnoses, and exclusion of BRCA1 and BRCA2 mutations have previously been described. ${ }^{4,15,16}$ As a functionally relevant BARD1 germline mutation was found in a patient with three primary tumors (breast, ovarian, and endometrial ${ }^{9}$ ), seven more patients with both breast and ovarian cancer were included in the analysis.

The frequency of the BARD1 Cys557Ser was evaluated in two large, independent series of breast cancer patients and healthy population controls originating from Southern and Central Finland. The first series included 888 unselected (described in detail in Syrjakoski et $a l^{17}$ and Kilpivaara $e a^{18}$ ) and 946 familial $^{16}$ breast cancer patients collected at the Helsinki University Central Hospital in Southern Finland. BRCA1 and BRCA2 mutations have been excluded in all the 403 patients with a strong family history of cancer (three or more first- or second-degree relatives with breast or ovarian cancer in the family, including the proband) as well as in 291 cases with one affected relative as previously described ${ }^{2,15,17}$ (also unpublished data). DNA from altogether 736 healthy female subjects collected at the same geographical region of Southern Finland was studied as healthy population controls. The second series where the frequency of the BARD1 Cys557Ser alteration was analyzed consists of 697 unselected breast cancer patients collected in Central Finland at Tampere University Hospital, Tampere, Finland $^{17}$ (also unpublished data). Of these, 442 patients have no family members affected with breast or ovarian cancer in addition to the proband, 183 have one, and 72 have two or more. BRCA1 and BRCA2 mutations have been excluded in 400 of the patients. ${ }^{17}$ Also, 358 healthy population controls from the same geographical region were genotyped for the BARD1 Cys557Ser alteration.

The frequency of the BARD1 variant Val507Met was studied in 888 unselected and in 710 familial breast cancer patients as well as in 736 population controls from the 
Helsinki series. As the variant has been associated with an elevation in postmenopausal breast cancer risk, the patients as well as the population controls were subgrouped according to their menopausal status (age 50 years was chosen as a surrogate for menopause: patients diagnosed $<50$ years were considered as premenopausal, and patients diagnosed $\geq 50$ years as postmenopausal).

Information on tumor characteristics (histology, grade, and size of the tumor; nodal status and distant metastasis; estrogen and progesterone receptor status) and age at breast cancer diagnosis was available from 1296 unselected breast cancer patients (altogether 1340 tumors). ${ }^{17,18}$ Written informed consent was obtained from each participating patient. This study has been performed with appropriate permissions from The Ministry of Social Affairs and Health in Finland as well as The Ethics Committees of the Departments of Obstetrics and Gynecology, and Oncology, in Helsinki University Central Hospital, Helsinki, and in the Tampere University Hospital, Tampere, Finland.

\section{Whole-gene mutation analysis and genotyping}

The mutation screening of the whole $B A R D 1$ coding region and exon-intron boundaries was carried out by conformation-sensitive gel electrophoresis (CSGE). The detection was based either on autoradiography, where one of the PCR primers was labeled with $\left[\gamma^{33} \mathrm{P}\right] \mathrm{dATP}$ (Amersham Biosciences, Uppsala, Sweden), or by staining the CSGE gels with silver nitrate (MERCK, Darmstadt, Germany). Primer sequences and PCR conditions used in CSGE analysis are available upon request. Samples with a band shift were reamplified, and the nucleotide changes were verified by direct sequencing using BigDye Terminator v3.1 Cycle Sequencing Kit and ABI 310 Sequencer (Applied Biosystems, Foster City, CA, USA).

The genotyping of DNA samples for the nt $1743 \mathrm{G} \rightarrow \mathrm{C}$ (Cys557Ser) and nt $1592 \mathrm{G} \rightarrow \mathrm{A}$ (Val507Met) variants in the Helsinki patient series and population controls was performed using Amplifluor ${ }^{\mathrm{TM}}$ fluorescent genotyping (K-Biosciences, Cambridge, UK). The genotyping for codon 557 was successful in 868/888 (98\%), 926/946 (98\%), and $725 / 736(99 \%)$ of the unselected and familial breast cancer patient and control samples, respectively, and 867/888 (98\%), 663/710 (93\%), and 718/736 (98\%) of the studied samples were successfully analyzed for codon 507, respectively. The genotypes of Tampere patients and healthy controls were determined by minisequencing. ${ }^{19}$

\section{Statistical analyses}

Associations between the BARD1 genetic alterations and breast cancer risk, as well as the tumor characteristics of the breast tumors, were tested by the $\chi^{2}$ test. The statistical analyses were carried out in the SPSS software (version 12.0 for Windows, SPSS, Chicago, IL, USA). All P-values were two-sided, and $P$-value $<0.01$ was considered statistically significant as suggested for the association studies for potential low-penetrance alleles. ${ }^{20}$

\section{Results and discussion}

Screening of the BARD1 gene for germline variants revealed seven coding sequence alterations (Table 1). The rare variants $1145 \mathrm{del} 21$ and Arg658Cys found in one family each $(1.9 \%)$ were also detected in $0.8 \%(8 / 974)$ and $1.9 \%$ (18/975) of healthy population controls, respectively (data not shown). The heterozygote carrier frequency of Arg378Ser was also similar in familial breast cancer patients $(40.4 \%, 21 / 52)$ and healthy population controls $(39.8 \%$, 97/244). These, as well as the silent variants Thr351Thr and His506His, have been described previously, and classified as polymorphisms with likely having no effect on cancer risk. 9, 13,14 Comprehensive analyses were carried out on the identified variants Cys557Ser and Val507Met that were recently suggested to associate with an increased breast cancer risk. These are described in more detail in the following.

The BARD1 cysteine 557 is a conserved amino acid that resides between the ankyrin repeats and the BRCT domain of a protein. This region has been shown to be essential for apoptosis induction and the interaction of BARD1 with a proto-oncogene $\mathrm{Bcl}-3 .^{21,22}$ The Cys557Ser alteration was recently suggested as a familial breast cancer allele in breast cancer families from Northern Finland. ${ }^{13}$ We found the variant here in one of the familial patients screened in the

Table 1 Observed coding region variants of the BARD1 gene

\begin{tabular}{|c|c|c|c|c|}
\hline Exon & Nt change & Effect on protein & Frequency for heterozygotes & Previously reported in reference \\
\hline 4 & $1126 \mathrm{G} \rightarrow \mathrm{C}$ & Thr351Thr & $17.3 \%(9 / 52)$ & 9,13 \\
\hline 4 & 1145 del 21 & 7 aa deletion & $1.9 \%(1 / 52)$ & 9,14 \\
\hline 4 & $1207 G \rightarrow C$ & Arg378Ser & $40.4 \%(21 / 52)$ & $13,14,23$ \\
\hline 6 & $1591 \mathrm{C} \rightarrow \mathrm{T}$ & His $506 \mathrm{His}$ & $7.7 \%(4 / 52)$ & 13,14 \\
\hline 6 & $1592 \mathrm{G} \rightarrow \mathrm{A}$ & Val507Met & $50 \%(26 / 52)$ & $9,13,14$ \\
\hline 7 & $1743 G \rightarrow C$ & Cys557Ser & $1.9 \%(1 / 52)$ & 9,13 \\
\hline 10 & $2045 \mathrm{C} \rightarrow \mathrm{T}$ & Arg658Cys & $1.9 \%(1 / 52)$ & 9,13 \\
\hline
\end{tabular}

$\mathrm{Nt}=$ nucleotide. 
initial analysis, and further studied it in two independent series of breast cancer patients and population controls. In the first study series, the Cys557Ser variant was observed with a low frequency both among unselected $(2.0 \%$, $17 / 868)$ and familial $(1.3 \%, 12 / 926)$ breast cancer patients. It was also present among healthy population controls with a frequency of $2.5 \%(19 / 725)$ (Table 2$)$. In the second independent series, the results were similar, with a frequencies of $2.4 \%(13 / 442)$ among unselected and $1.6 \%$ (4/255) among familial breast cancer patients, and $2.2 \%$ (8/358) among healthy controls (Table 2). Breast cancer patients were further divided into subgroups based on the number of affected relatives and whether or not there were ovarian cancer patients in the family (Table 2). No evidence for elevated breast cancer risk of the BARD1 Cys557Ser variant was observed in any of the study cohorts or subgroups; indeed, the variant frequency was slightly lower among the familial patient series than among population controls. No association between the variant and any of the tumor characteristics (tumor histology, grade, and size; nodal status and distant metastasis; estrogen and progesterone receptor status) was seen either (data not shown), and the mean age at breast cancer diagnosis was similar between the carriers and noncarriers of the variant (57.3 and 58.2 years, respectively $(P=0.723))$.

Previously, the Cys557Ser variant has been observed in two sporadic Caucasian breast/uterine/ovarian tumors from the US population, ${ }^{9}$ and in one Italian breast/ovarian cancer family. ${ }^{23}$ In the former study, the variant was classified as a polymorphism and in the latter, possibly cancer related. Association analysis to evaluate breast cancer risk was not possible in either study. In another recent study, the variant was found in seven out of 126 (5.6\%) breast cancer families from Northern Finland and in 14 out of 1018 (1.4\%) healthy population controls, suggesting that the carriers would have a four-fold increased risk for familial breast cancer. ${ }^{13}$ The prevalence was highest among patients whose family history included only breast and not ovarian cancer (7/94, 7.4\% vs 14/1018, 1.4\%; OR 5.8; 95\% CI 2.3-14.7, $P=0.001)$. Our results with two extensive and independent series of breast cancer patients do not support association of Cys557Ser with breast cancer risk either among familial or unselected breast cancer patients. We found the variant altogether in $1.4 \%$ of 1181 familial breast cancer patients, in $2.2 \%$ of 1565 unselected breast cancer patients, and in $2.5 \%$ of 1083 healthy controls, respectively, and there was no difference in the frequencies by different family history. The discrepancy between the present study and the study by Karppinen et $a l^{13}$ (2004) could be due to the small sample size in the latter, which may have resulted in a positive association by chance. Differences in the population stratification of the breast cancer families and population controls used could also be a possible cause 
Table 3 Genotypes of the BARD1 Val507Met in 867 unselected and 663 familial breast cancer patients, and in 718 healthy controls

\begin{tabular}{|c|c|c|c|c|c|c|c|}
\hline & \multicolumn{2}{|c|}{ Cases } & \multicolumn{2}{|c|}{ Controls } & \multirow[b]{2}{*}{$O R^{\mathrm{a}}$} & \multirow[b]{2}{*}{$95 \% \mathrm{Cl}$} & \multirow[b]{2}{*}{$P$} \\
\hline & $n$ & $\%$ & $n$ & $\%$ & & & \\
\hline $\begin{array}{l}\text { Unselected breast cancer patients, all }(n=867) \\
\text { AA } \\
\text { AG } \\
\text { GG } \\
\text { GG+AG }\end{array}$ & $\begin{array}{l}178 \\
448 \\
241 \\
689\end{array}$ & $\begin{array}{l}20.5 \\
51.7 \\
27.8 \\
79.5\end{array}$ & $\begin{array}{l}718 \\
179 \\
355 \\
184 \\
539\end{array}$ & $\begin{array}{l}24.9 \\
49.4 \\
25.6 \\
75.1\end{array}$ & $\begin{array}{l}1 \\
1.27 \\
1.32 \\
1.29\end{array}$ & $\begin{array}{l}0.99-1.63 \\
0.99-1.75 \\
1.02-1.63\end{array}$ & $\begin{array}{l}0.061 \\
0.056 \\
0.037\end{array}$ \\
\hline $\begin{array}{l}\text { Unselected breast cancer patients, diagnosis } \geq 50 \text { years }(n=591) \\
\text { AA } \\
\text { AG } \\
\text { GG } \\
\text { GG+AG }\end{array}$ & $\begin{array}{l}126 \\
310 \\
155 \\
465\end{array}$ & $\begin{array}{l}21.7 \\
52.4 \\
26.2 \\
79.7\end{array}$ & $\begin{array}{r}250 \\
60 \\
128 \\
62 \\
190\end{array}$ & $\begin{array}{l}24.0 \\
51.2 \\
24.8 \\
76.0\end{array}$ & $\begin{array}{l}1 \\
1.15 \\
1.19 \\
1.17\end{array}$ & $\begin{array}{l}0.80-1.67 \\
0.78-1.82 \\
0.82-1.66\end{array}$ & $\begin{array}{l}0.450 \\
0.421 \\
0.392\end{array}$ \\
\hline
\end{tabular}

A codes for Met and $G$ codes for Val.

${ }^{a}$ Compared to AA genotype.

for a chance association. However, the possibility that the Cys557Ser variant interacts with another, unknown risk allele in the breast cancer families from Northern Finland, but not in Central or Southern Finland, cannot be excluded.

The Met-allele of the common BARD1 missense substitution Val507Met has been suggested to associate with an increased breast cancer risk in postmenopausal women in a study including 73 pre- and 70 postmenopausal breast cancer patients and 152 healthy controls (adjusted OR 2.05; 95\% CI 1.01-4.16). ${ }^{14}$ The residue resides within the conserved ankyrin repeat domain, which is responsible for the protein-protein interactions. The particular codon is, however, not fully conserved.

The BARD1 Val507Met was found to be very common also in our study population. The allele frequencies for $G$ (coding for Val) and A (coding for Met) were 0.54 and 0.46 among the unselected breast cancer patients, 0.51 and 0.49 among the familial breast cancer patients, and 0.50 and 0.50 among the healthy population controls, respectively. The Val-allele (either in homo- or heterozygous form) was slightly more common among unselected breast cancer patients than among healthy controls (OR 1.29; 95\% CI 1.02-1.63; $P=0.037$; Table 3). The difference in frequency was highest for the Val-homozygotes, although the statistical significance was not reached (Table 2). When the patients were subgrouped according to their menopausal status, the higher Val-allele carrier frequency was found in the pre- (OR 1.47; 95\% CI 1.018-2.12; $P=0.039)$ but not in the postmenopausal women (OR 1.17; 95\% CI 0.821.66; $P=0.392$ ), when compared to Met-homozygotes. Similar to the whole cohort, the difference in frequency was highest for the Val-homozygotes (OR 1.61; 95\% CI $1.05-2.47 ; P=0.028)$. Results from the three-value trend test suggested a slightly elevated cancer risk for the Valallele carriers in the premonopausal patient group but not in the whole cohort of unselected patients (data not shown). The age at breast cancer diagnosis did not differ between the different genotype carriers (55.8 years for the Met-homozygotes, 56.2 years for the heterozygotes, and 55.9 years for the Val-homozygotes). No association between the Val507Met and any of the tumor characteristics or cumulative 5-year survival among the unselected patients was observed (data not shown). Also, no increase in breast cancer risk was observed in familial breast cancer patients. Altogether, our results suggest that this variant does not have an effect on familial breast cancer susceptibility, whereas some evidence for a modest elevation in breast cancer risk among unselected breast cancer patients, especially among those below 50 years, was obtained.

In summary, rarity or absence of clearly pathologic $B A R D 1$ mutations in this study and previous studies suggests that BARD1 is not a major gene underlying familial breast cancer. Our results also indicate that neither the BARD1 Cys557Ser nor Val507Met variants predispose to familial breast cancer. 


\section{Acknowledgements}

We thank Dr Päivi Heikkilä for her help with tumor data, Minna Merikivi, Nina Puolakka, Päivi Virtanen, and Kristiina Selkee for patient contacts, and Kati Rouhento and Johanna Tommiska for technical assistance. The Finnish Cancer Registry is gratefully acknowledged for cancer data. This study was financially supported by the Helsinki University Central Hospital Research Fund, Finnish Academy of Sciences, Finnish Cancer Society, Sigrid Juselius Foundation, Foundation of the Finnish Cancer Institute, Maud Kuistila Foundation, and the Medical Research Fund of Tampere University Hospital.

\section{References}

1 Ford D, Easton DF, Stratton $\mathrm{M}$ et al: Genetic heterogeneity and penetrance analysis of the BRCA1 and BRCA2 genes in breast cancer families. The Breast Cancer Linkage Consortium. Am J Hum Genet 1998; 62: 676-689.

2 Vahteristo P, Eerola H, Tamminen A, Blomqvist C, Nevanlinna H: A probability model for predicting BRCA1 and BRCA2 mutations in breast and breast-ovarian cancer families. Br J Cancer 2001; 84: 704-708.

3 Meijers-Heijboer $\mathrm{H}$, van den Ouweland A, Klijn J et al: Lowpenetrance susceptibility to breast cancer due to CHEK2(*)1100delC in noncarriers of BRCA1 or BRCA2 mutations. Nat Genet 2002; 31: 55-59.

4 Vahteristo P, Bartkova J, Eerola $\mathrm{H}$ et al: A CHEK2 genetic variant contributing to a substantial fraction of familial breast cancer. $\mathrm{Am}$ I Hum Genet 2002; 71: 432-438.

5 Kilpivaara O, Vahteristo P, Falck J et al: CHEK2 variant I157T may be associated with increased breast cancer risk. Int J Cancer 2004; 111: $543-547$

6 Antoniou AC, Pharoah PD, McMullan G, Day NE, Ponder BA, Easton D: Evidence for further breast cancer susceptibility genes in addition to BRCA1 and BRCA2 in a population-based study. Genet Epidemiol 2001; 21: 1-18.

7 Antoniou AC, Pharoah PD, McMullan G et al: A comprehensive model for familial breast cancer incorporating BRCA1, BRCA2 and other genes. Br J Cancer 2002; 86: 76-83.

$8 \mathrm{Wu}$ LC, Wang ZW, Tsan JT et al: Identification of a RING protein that can interact in vivo with the BRCA1 gene product. Nat Genet 1996; 14: 430-440.

9 Thai TH, Du F, Tsan JT et al: Mutations in the BRCA1-associated RING domain (BARD1) gene in primary breast, ovarian and uterine cancers. Hum Mol Genet 1998; 7: 195-202.
10 Kleiman FE, Manley JL: The BARD1-CstF-50 interaction links mRNA 3' end formation to DNA damage and tumor suppression. Cell 2001; 104: 743-753.

11 Irminger-Finger I, Leung WC, Li J et al: Identification of BARD1 as mediator between proapoptotic stress and p53-dependent apoptosis. Mol Cell 2001; 8: 1255-1266.

12 Yoshikawa K, Ogawa T, Baer R et al: Abnormal expression of BRCA1 and BRCA1-interactive DNA-repair proteins in breast carcinomas. Int J Cancer 2000; 88: 28-36.

13 Karppinen SM, Heikkinen K, Rapakko K, Winqvist R: Mutation screening of the BARD1 gene: evidence for involvement of the Cys557Ser allele in hereditary susceptibility to breast cancer. J Med Genet 2004; 41: e114.

14 Ishitobi M, Miyoshi Y, Hasegawa S et al: Mutational analysis of BARD1 in familial breast cancer patients in Japan. Cancer Lett 2003; 200: $1-7$.

15 Vehmanen P, Friedman LS, Eerola $\mathrm{H}$ et al: Low proportion of BRCA1 and BRCA2 mutations in Finnish breast cancer families: evidence for additional susceptibility genes. Hum Mol Genet 1997; 6: $2309-2315$.

16 Eerola H, Blomqvist C, Pukkala E, Pyrhonen S, Nevanlinna $\mathrm{H}$ Familial breast cancer in southern Finland: how prevalent are breast cancer families and can we trust the family history reported by patients? Eur J Cancer 2000; 36: 1143-1148.

17 Syrjakoski K, Vahteristo P, Eerola $\mathrm{H}$ et al: Population-based study of BRCA1 and BRCA2 mutations in 1035 unselected Finnish breast cancer patients. J Natl Cancer Inst 2000; 92: 1529-1531.

18 Kilpivaara O, Bartkova J, Eerola $\mathrm{H}$ et al: Correlation of CHEK2 protein expression and c.1100delC mutation status with tumor characteristics among unselected breast cancer patients. Int $J$ Cancer 2005; 113: 575-580

19 Syvanen AC, Sajantila A, Lukka M: Identification of individuals by analysis of biallelic DNA markers, using PCR and solid-phase minisequencing. Am J Hum Genet 1993; 52: 46-59.

20 Houlston RS, Peto J: The future of association studies of common cancers. Hum Genet 2003; 112: 434-435.

21 Dechend R, Hirano F, Lehmann K et al: The Bcl-3 oncoprotein acts as a bridging factor between NF-kappaB/Rel and nuclear co-regulators. Oncogene 1999; 18: 3316-3323.

22 Jefford CE, Feki A, Harb J, Krause KH, Irminger-Finger I: Nuclearcytoplasmic translocation of BARD1 is linked to its apoptotic activity. Oncogene 2004; 23: 3509-3520.

23 Ghimenti C, Sensi E, Presciuttini S et al: Germline mutations of the BRCA1-associated ring domain (BARD1) gene in breast and breast/ovarian families negative for BRCA1 and BRCA2 alterations. Genes Chromosomes Cancer 2002; 33: 235-242. 